\title{
BMJ Open Effects of priming intermittent theta burst stimulation on upper limb motor recovery after stroke: study protocol for a proof-of-concept randomised controlled trial
}

To cite: Zhang JJ, Fong KNK. Effects of priming intermittent theta burst stimulation on upper limb motor recovery after stroke: study protocol for a proof-of-concept randomised controlled trial. BMJ Open 2020;10:e035348. doi:10.1136/ bmjopen-2019-035348

- Prepublication history and additional material for this paper are available online. To view these files, please visit the journal online (http://dx.doi. org/10.1136/bmjopen-2019035348).

Received 31 October 2019 Revised 11 February 2020 Accepted 19 February 2020

Check for updates

(c) Author(s) (or their employer(s)) 2020. Re-use permitted under CC BY-NC. No commercial re-use. See rights and permissions. Published by BMJ.

Department of Rehabilitation Sciences, The Hong Kong Polytechnic University, Hong Kong SAR, China

Correspondence to

Jack Jiaqi Zhang;

17902718r@connect.polyu.hk

\section{ABSTRACT}

Introduction Intermittent theta burst stimulation (iTBS), a form of repetitive transcranial magnetic stimulation (rTMS), delivered to the ipsilesional primary motor cortex (M1), appears to enhance the brain's response to rehabilitative training in patients with stroke. However, its clinical utility is highly subject to variability in different protocols. New evidence has reported that preceding iTBS, with continuous theta burst stimulation (CTBS) may stabilise and even boost the facilitatory effect of iTBS on the stimulated M1, via metaplasticity. The aim of this study is to investigate the effects of iTBS primed with cTBS (ie, priming iTBS), in addition to robot-assisted training (RAT), on the improvement of the hemiparetic upper limb functions of stroke patients and to explore potential sensorimotor neuroplasticity using electroencephalography (EEG).

Methods and analysis A three-arm, subjects and assessors-blinded, randomised controlled trial will be performed with patients with chronic stroke. An estimated sample of 36 patients will be needed based on the prior sample size calculation. All participants will be randomly allocated to receive 10 sessions of rTMS with different TBS protocols (cTBS+iTBS, sham cTBS+iTBS and sham cTBS+sham iTBS), three to five sessions per week, for $2-3$ weeks. All participants will receive 60 min of RAT after each stimulation session. Primary outcomes will be assessed using Fugl-Meyer Assessment-Upper Extremity scores and Action Research Arm Test. Secondary outcomes will be assessed using kinematic outcomes generated during RAT and EEG.

Ethics and dissemination Ethical approval has been obtained from The Human Subjects Ethics Subcommittee, University Research Committee of The Hong Kong Polytechnic University (reference number: HSEARS20190718003). The results yielded from this study will be presented at international conferences and sent to a peer-review journal to be considered for publication. Trial registration number NCT04034069.

\section{INTRODUCTION}

Repetitive transcranial magnetic stimulation (rTMS) has been extensively investigated as an add-on form of therapy for stroke
Strengths and limitations of this study

- This study will be the first randomised controlled trial to explore the effects of priming intermittent theta burst stimulation (iTBS) in regard to facilitating hemiparetic upper limb recovery in patients with stroke.

- This study investigates sensorimotor desynchronisation along with the improvement of upper limb functions, in association with priming iTBS.

- The study attempts to potentiate the brain response to iTBS by using an inhibitory priming session.

- This study contributes to the optimal use of TBS in poststroke upper limb rehabilitation.

- This study has limited generalisability to stroke patients at the acute phase.

rehabilitation. ${ }^{1}$ rTMS is usually limited to frequencies of $20 \mathrm{~Hz}$ or less, due to safety concerns, in human studies. ${ }^{2}$ However, in animal studies, effects on synaptic plasticity are usually induced by repeated short bursts of high-frequency $(50 \mathrm{~Hz})$ stimulation, given at a frequency from 3 to $5 \mathrm{~Hz}$ and known as theta burst stimulation (TBS). ${ }^{3}$ Huang et al were the first to investigate the neurophysiological effects of TBS, delivered via a magnetic stimulator, in the human primary motor cortex (M1) and demonstrated that 600-pulse intermittent theta burst stimulation (iTBS) enhanced corticomotor excitability in healthy human subjects, whereas 600-pulse continuous theta burst stimulation (cTBS) did the opposite. ${ }^{4}$ Serial TBS sessions delivered at a relatively low intensity were subsequently investigated in stroke survivors and safety concerns regarding TBS in this population appear to be minor and rare.$^{5-8}$ Various experiments with humans have also demonstrated that TBS is able to induce neuroplastic changes of the stimulated M1 in a 
relatively short conditioning period (ie, $40 \mathrm{~s}$ for standard 600-pulse cTBS and 3 min for standard 600-pulse iTBS), ${ }^{9}$ thus reducing the time spent receiving treatment.

A substantial number of clinical trials with stroke patients have revealed that iTBS of the ipsilesional M1 significantly improves hemiplegic arm ${ }^{56}{ }^{10-12}$ and hand ${ }^{8}$ motor functions, compared with sham stimulations. Similar effects have also been observed in studies using cTBS of the contralesional M1. ${ }^{13}{ }^{14}$ However, some trials have not shown any additional benefits on upper limb motor outcomes from iTBS or cTBS in stroke survivors, in contrast to sham TBS. ${ }^{713} \mathrm{~A}$ recent meta-analysis showed that a pooled standardised effect size of iTBS was 0.60 , while that of cTBS was 0.35 for upper limb motor outcomes in patients with stroke, ${ }^{15}$ indicating that the increment of the excitability of the affected M1 through iTBS is critical for improving the brain's response to motor training in patients with stroke. However, substantial response variability regarding iTBS among humans may contribute to the use of different protocols among current studies, ${ }^{16}{ }^{17}$ which limits their clinical utility.

It has been shown that the history of neuronal activities is one of the major factors that could influence the brain's response to TBS. $^{18}$ Synaptic plasticity is regulated by previous neuronal activities via metaplasticity. Metaplasticity is a neuroprotective mechanism that modulates the threshold of synaptic plasticity to ensure that the neural system cannot be predominated by long-term potentiation or long-term depression. ${ }^{19}$ Excitatory rTMS over the M1 may be unable to facilitate corticomotor excitability when the neuronal activities have already been elevated before stimulation, which is likely happening when patients with stroke receive extensive training before noninvasive brain stimulation.

Considering the mechanism of metaplasticity, several priming stimulation protocols, designed to incorporate a priming session followed by a stimulation session, have been investigated with healthy individuals. ${ }^{20}$ An inhibitory priming stimulation via cTBS may ensure or even boost the facilitatory effect of subsequent excitatory stimulation sessions via iTBS. In healthy individuals, this priming protocol seems to amplify the facilitatory effect of excitatory stimulation, compared with iTBS alone, as reflected by the increased amplitude of motor evoked potential (MEP) ${ }^{21-23}$ Metaplasticity is also significantly involved in rTMS studies for patients with neuropsychiatric disorders. ${ }^{24}{ }^{25}$ However, to date no study has investigated the effects of priming iTBS protocols in patients with stroke.

Various neurological biomarkers of stroke motor recovery have been proposed. ${ }^{26}$ Electroencephalography (EEG), a non-invasive measure of cortical neuronal oscillation, is of great interest, because it is a relatively convenient and well-tolerated neurophysiological technique for patients with stroke. Sensorimotor event-related desynchronisation (ERD), a neurophysiological marker of sensorimotor activation, could be induced through either action observation or action execution. ${ }^{27}$ Previously, attention has been paid to movement-related sensorimotor ERD, which has been shown to be correlated with the severity of hemiplegia in patients with stroke. ${ }^{28} 29$ Subsequently, researchers began to investigate sensorimotor ERD induced by observing mirror visual feedback (MVF) in healthy adults and patients with stroke. ${ }^{3031} \mathrm{~A}$ pilot study has demonstrated that multiple sessions of iTBS appear to enhance MVF-induced sensorimotor ERD in healthy adults. ${ }^{32}$ So far, MVF-induced sensorimotor ERD has not been used as an outcome of neuroplasticity in any clinical stroke trial in order to examine its potential as a biomarker for stroke motor recovery. Sensorimotor ERD will be used to probe cortical oscillatory activities of large number of neurons in different rhythms, during a given task (movement or movement observation). A previous study comparing the effects of TBS on MEPs and movement-related rhythmic oscillations showed that the modulatory effect of TBS was more reliable on movement-related ERD than that on MEPs. ${ }^{33}$ The potential explanations may be that TMS-based metrics may not represent all cortical responses, reflecting exclusively those destined to the spinal cord,$^{33}$ and the magnitude of TMS-based metrics is also contaminated by the neuronal responses at subcortical and spinal levels, as well as the peripheral $\mathrm{MEP}^{34}$ when a suprathreshold stimulation intensity is used for the measurements. Hence, we decide to use sensorimotor desynchronisation in this study, which may provide new insight about the sensorimotor neuroplasticity in association with priming iTBS.

Therefore, our study has two objectives. First, we investigate the effects of 10 sessions of rTMS using different TBS protocols (ie, cTBS+iTBS, sham cTBS+iTBS and sham cTBS+sham iTBS), in addition to standard robot-assisted training (RAT) for both the proximal and distal joints of the hemiparetic upper limb, delivered across three to five sessions per week for 2-3 weeks, on improving the hemiparetic upper limb functions of stroke survivors. FuglMeyer Assessment-Upper Extremity (FMA-UE) scores and Action Research Arm Test (ARAT) will be used as the primary outcome measures. Safety profiles will be systematically collected during each session of the intervention, using a standard questionnaire. Second, we investigate the effects of different TBS protocols, cTBS+iTBS, sham cTBS+iTBS and sham cTBS+sham iTBS, in addition to RAT, on upper limb kinematic outcomes yielded from each RAT session, and sensorimotor ERD induced by hemiparetic hand movement and observation of the MVF of non-paretic hand movement, in patients with stroke.

\section{METHODS}

This study protocol has been written according to the Standard Protocol Items for Randomised Trials statement. ${ }^{35}$

\section{Study design}

This study is designed as a three-arm, parallel group, subjects-blinded and assessors-blinded, sham-controlled randomised controlled trial (RCT). Potential participants with stroke will be recruited through convenience 
sampling from self-support groups in the community in Hong Kong. The study will be conducted in a local university laboratory.

\section{Inclusion and exclusion criteria}

Participants must meet all of the following criteria: (1) have a diagnosis of a unilateral ischaemic or haemorrhagic first-ever stroke; (2) time after stroke onset $\geq 6$ months ${ }^{36}$; (3) between 18 and 64 years old; (4) reside in community dwellings; (5) with residual upper limb impairment $\geq$ second level in the Functional Test for the Hemiplegic Upper Extremity (FTHUE) ${ }^{37}$ FTHUE is a fast screening tool for upper limb functional movement, which has been used as a screening in our previous RCTs. ${ }^{38}{ }^{39}$ FTHUE levels two to four are defined as low upper limb functioning poststroke, and levels five and seven are defined as high upper limb functioning poststroke ${ }^{38}$; (6) able to understand simple verbal instruction and follow one-step commands; (7) able to give informed written consent to participate in the study.

Although TBS is often regarded as safe for certain subjects, the greatest acute risk of TMS is the rare occurrence of induced seizures. Besides seizures, other risks include minor pain, such as a headache or local discomfort, minor cognitive changes and psychiatric symptoms. In this study, patients who meet any of the following rTMS contraindications will not be included: (1) unstable medical condition; (2) history of epileptic seizures, unconsciousness or intracranial hypertension; (3) serious heart disease; (4) pregnancy; (5) with metal implants in vivo, such as a pacemaker, artificial cochlear or implant brain stimulator; (6) history of receiving a craniotomy; (7) taking any centrally acting drugs in the recent 3 months. ${ }^{2}$ To ensure safety, the participants will be under the supervision of at least one investigator who has completed training in TMS. All participants will undergo a safety screening for the potential risks of TMS to ensure they are eligible to participate in this study. ${ }^{2}$

In addition to TMS contraindications, participants who meet any of the following criteria will be also excluded: (1) previous diagnosis of any neurological disease excluding stroke; (2) presence of any sign of cognitive problems (Abbreviated Mental Test, Hong Kong Cantonese version $<6 / 10)^{40}$; (3) patients with extreme spasticity over the elbow or wrist in the hemiparetic upper limb (Modified Ashworth score $>2$ ), ${ }^{41}$ or severe pain that hinders upper limb movement; (4) other notable impairments of the upper limb not affected by stroke (eg, a recent fracture, severe osteoarthritis, congenital upper limb deformity); (5) significant aphasia or difficulty understanding the instructions given by the investigators; (6) any sign of anxiety and/or depression screened by the Hospital Anxiety and Depression Scale, using a cut-off value of 8 in both subscales ${ }^{42}$; (7) concurrent participation in upper limb rehabilitation training in a hospital, university laboratory or other rehabilitation settings, or active participation in another clinical trial.

\section{Sample size estimation}

Since the difference among the effects of priming iTBS in hemiparetic upper limb training has not been previously investigated, we have estimated the sample size based on current studies comparing iTBS and sham stimulation. A recent meta-analysis yields a pooled Cohen's $d$ of 0.60 for a two-group design in favour of iTBS improving upper limb motor outcomes, in contrast to sham stimulation. ${ }^{15}$ An effect size (d) of 0.60 corresponds approximately to an effect size (f) of 0.30 for a study design of threegroup comparisons. An estimate of sample size for each group in a three-group design, given a power of 0.80 and a two-tailed alpha error probability of 0.05 , is 27 patients in total. When considering the drop-out rate of $20 \%$, we therefore plan to recruit 12 participants for each group (a total of 36) for this study.

\section{Randomisation}

Three parallel groups will be employed: (1) cTBS+iTBS; (2) sham cTBS+iTBS; (3) sham cTBS+sham iTBS. The collection of demographic characteristics (age, gender, education, side of hemiplegia, handedness, type of stroke, time from onset to treatment, lesion site(s)) and baseline assessments will be performed prior to randomisation. Participants' medical information related to their stroke will be retrieved from the electronic clinical management system in the hospital after receiving consent. All participants will be randomly allocated in a 1:1:1 ratio to each group after the screening and baseline assessments have been carried out. A random sequence will be generated using Minimise software. ${ }^{43}$ Participants will be pre-stratified based on their hemiparetic upper limb functioning (ie, FTHUE high functioning vs low functioning). The allocation sequence will be concealed from all investigators and assessors. Participants will receive 10 sessions of TBS intervention combined with RAT, 3-5 sessions per week, for 2-3weeks. We decide to adopt a more flexible training schedule, because most community stroke survivors are unable to visit our laboratory on a daily basis. Similar schedule for motor training has been used in previous studies for patients with chronic stroke. ${ }^{445}$

\section{Intervention}

\section{TBS session}

A total of 10 sessions of TBS will be delivered using MagPro magnetic stimulators (MagVenture, Denmark) connected with a figure-of-eight coil. Resting motor threshold (RMT) is defined as the minimum stimulation intensity over the hot spot that could elicit a MEP of no less than $50 \mu \mathrm{V}$ in 5 out of 10 trials over the contralesional first dorsal interosseous muscle. The stimulation point is the hotspot mirrored over the midsagittal line (ie, ipsilesional M1), verified and maintained by a TMS-navigation system (Localite, Bonn, Germany).

We follow the standard 600-pulse TBS protocol proposed by Huang and Rothwell ${ }^{4}$ : iTBS: 20 trains of 10 bursts given with eight-second intervals, with a total of 600 pulses, around 3 min per session; cTBS: 20 trains of 10 
bursts given with $0.2 \mathrm{~s}$ intervals, with a total of 600 pulses, around $40 \mathrm{~s}$ per session. All stimulations will be delivered over the ipsilesional M1. The intensity of the TBS will be set at $70 \%$ RMT. Sham cTBS will be delivered with the same coil, but the intensity will be reduced to $20 \%$ of the individual RMT. Intensity reduction has been used as sham stimulation in some previous clinical studies ${ }^{546}$ and our pilot study. ${ }^{32}$ The interval between the priming session and the conditioning session will be 10 min..$^{21}$ All participants will be informed that TBS is delivered in a subthreshold intensity that cannot induce significant limb movement or somatosensory perception.

\section{Robot-assisted training}

Participants will be required to undergo two forms of RAT for the proximal and distal joints of the hemiparetic upper limb, respectively, after each TBS session. RAT will commence 5 min after the completion of the TBS session. ${ }^{11}$ A Fourier M2 robot (Fourier Intelligence Company, Shanghai, China) will be used for the upper limb proximal joint training. The Fourier M2 robot is an end-effector robot-assisted upper limb rehabilitation device, supported by tailored interactive television games in the device. A HandyRehab hand robot (Zunosaki Company, Hong Kong SAR, China) will be used for upper limb distal joint training. The device provides power-driven extension and grasping force to the fingers and thumb in order to assist the patient with opening and closing the paretic hand by means of surface electromyography (EMG) triggered from the signals through the forearm extensors and flexors. Active and passive modes are available in both robots. Whenever patients are unable to use the active modes due to the severity of the upper limb hemiplegia, passive modes will be used.

\section{Proximal joint training}

The Fourier M2 robot targets: (1) flexion and extension of the shoulder joint; (2) flexion and extension of the elbow; (3) internal and external rotation of the shoulder joint; (4) abduction and adduction of the shoulder joint. Before each training session, the size of the maximal active range of motion (ROM) for the hemiplegic upper limb will be assessed for each participant. Two-minute warm-up sessions will be delivered before and after each training session, in which participants will receive passive-mode RAT to mobilise the paretic upper limb. The movement trajectory will be predefined as a square and its size will be calculated based on participants' maximal active ROM. In the training session, the participants will be asked to move their hemiparetic upper limb to reach sequentially presented targets in an interactive game. Each proximal joint training session will last for around $30 \mathrm{~min}$, with a break of 5-10 min (see figure 1A for a demonstration of proximal joint RAT). The assistive mode will be used to train the patients with limited voluntary shoulder and elbow movement (ie, the patient initiates the movement and the robot then produces assistive force according to the subject's effort). For patients who cannot initiate

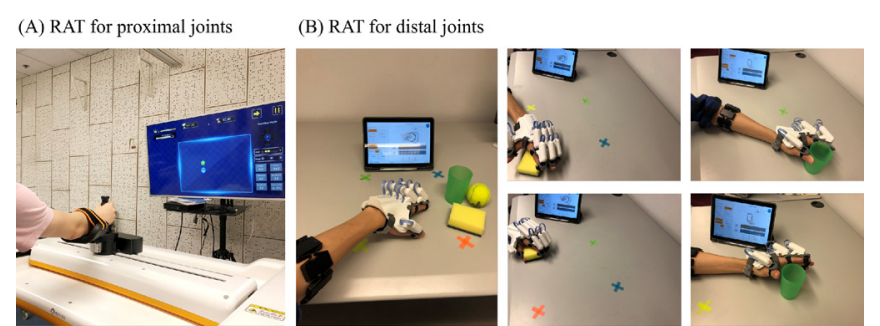

Figure 1 A demonstration of RAT. Note: the persons depicted are not patient and were taken with the participants knowledge. RAT, robot-assisted training.

movement by themselves, the passive mode will be used. The active mode and resistive mode will be used to train the patients with voluntary shoulder and elbow movement. Each participant has to sit in front of the robot with a computer screen attached to the device. The participant will wear a trunk-fixed belt to minimise compensatory movement of the trunk during training.

\section{Distal joint training}

The HandyRehab hand robot is lightweight, powered by lithium batteries and allows the subject to perform a full hand grasp/release movement in either the spherical grip or cylindrical grip mode. The EMG trigger threshold will be adjusted based on patients' hemiplegic arm function. Participants will be instructed to perform the different types of hand movements to pick up an object (ie, a ball, sponge or a cup) on a table, move it vertically and/or horizontally to four predefined targets and release the object. The distance between the targets and participants will be adjusted based on their active ROM of proximal joints in the paretic upper limb. The EMG-triggered level will be adjusted based on patients' ability (ie, active mode) and gradually increased as the training progresses. For patients without any detectable EMG signal from the paretic forearm, the passive mode will be used. Each distal joint training session will last for around $30 \mathrm{~min}$, with a break of 5-10 min (see figure 1B for a demonstration of distal joint RAT). In total, each RAT session lasts for approximately $60 \mathrm{~min}$ ( $30 \mathrm{~min}$ for proximal joints and 30 min for distal joints), with 10 sessions in total. An investigator with a background in physiotherapy or occupational therapy will supervise each participant during each robot training session to ensure the correct positioning is used and that and participants become familiar with the training.

\section{Outcome measurements \\ Primary outcomes}

The FMA-UE and ARAT will be used as the primary outcomes for this study. ${ }^{47}$ The FMA-UE is a clinical assessment for upper limb motor impairment after stroke. It includes 33 items assessing the movement, coordination and reflex actions of the shoulder, elbow, forearm and wrist, and the hand joints of the paretic arm. Each item consists of a three-point scale $(0,1$ and 2), with a total maximum score of 66 . The minimal clinically important 
difference (MCID) of the FMA-UE is 5.25 points. ${ }^{48}$ The FMA-UE will be conducted at four time points: baseline, mid-term (ie, after five sessions), post-training (ie, after 10 sessions) and follow-up (ie, 2 weeks after the completion of all training sessions). An assessor who is unaware of the treatment allocation will carry out the assessment for each participant. The ARAT is a clinical assessment for upper limb functional activities for patients with stroke. The ARAT assesses proximal and distal components of upper limb function. It consists of four subscales: grasp, grip, pinch and gross movement. It has 19 movement tasks, each graded using a four-point scale (total scores range from 0 to 57 ). The MCID of ARAT is 5.70 points. ${ }^{49}$ ARAT will be conducted at the same four time points as the FMA-UE. An assessor who is unaware of the treatment allocation will carry out the assessment for each participant. Assessors will be trained and tested by the principle investigator, before conducting clinical assessments.

\section{Secondary outcomes}

Kinematic metrics generated during each session of RAT will be used as secondary outcomes for the participants' upper limb function. The following kinematic metrics retrieved from the M2 robot will be used as the upper limb motor outcomes in a further analysis: (1) the size of the maximal active ROM; (2) the mean velocity of movement during the training session; (3) the movement trajectory during the training session. Movement trajectory will be further calculated as the hand-path ratio, which is defined as the real distance divided by the shortest distance between object targets. ${ }^{50}$

In order to investigate the potential neuroplasticity elicited by the training, we will invite patients to participate in EEG examinations. We expect that around five patients from each group will take part in the EEG examinations before and after the intervention. Kinematic and EEG outcomes will be assessed in a non-blinded manner (see figure 2 for a flowchart).

\section{EEG acquisition}

EEG will be captured with a 64-channel cap using a digital DC EEG amplifier. Electrode impedance will be kept below $10 \mathrm{k} \Omega$ and the signal will be sampled at $1000 \mathrm{~Hz}$. Movement-related ERD and MVF-induced ERD will be evaluated in this study. For movement-related ERD, participants will be asked to perform finger taps three times (or attempt to move their finger if they cannot perform the movement fluently) on a computer keyboard with the index finger of their unaffected side, in response to auditory cues (ie, a $300 \mathrm{~ms}$ beep sound) delivered at random intervals (from $7 \mathrm{~s}$ to $10 \mathrm{~s}$ ), and to relax their hand after the completion of the movement.

For MVF-induced ERD, participants will be asked to perform finger taps three times on a computer keyboard with the index finger of their unaffected side, in response to auditory cues delivered at random intervals (from 7 $\mathrm{s}$ to $10 \mathrm{~s}),{ }^{51}$ and to relax their hand after the completion of the movement. A widely used EEG paradigm exploring the effects of MVF will be utilised in the present study $^{30-325152}$; movements will be performed under two conditions. (1) MVF of the hand movement: participants will be required to perform unilateral finger tapping while viewing MVF. MVF will be created using a physical mirror $(406 \times 432 \mathrm{~mm})$ placed over their midsagittal plane, between both arms. (2) Direct visual feedback of the hand movement: participants will be required to perform unilateral finger tapping while directly looking at their moving finger. The affected hand will be hidden by a non-reflective board.

The order of conditions will be allocated randomly by drawing lots. A total of 60 movements will be collected for each condition (affected index movement, unaffected index with mirror view and unaffected index with direct view), with 180 movements in total.

\section{EEG preprocessing}

Raw EEG signals will be band-pass filtered between 1 and $80 \mathrm{~Hz}$ and then down-sampled at $250 \mathrm{~Hz}$. Additionally, a $50 \mathrm{~Hz}$ notch filter will be applied. Data will be offline rereferenced to bilateral mastoid electrodes. Signals with significant movement artefacts and long-term eye closure will be rejected during a visual inspection. Subsequently, EEG will be segmented in $7000 \mathrm{~ms}$ epochs (prestimulus $-3000 \mathrm{~ms}$ and poststimulus $4000 \mathrm{~ms}$, with 0 as the first finger tap). Eye movement artefacts will be corrected using an independent component analysis algorithm. ${ }^{53}$ Typical components reflecting the eye blinks and horizontal movements will then be rejected.

\section{EEG time-frequency analysis}

Clean epochs will be analysed in a time-frequency domain. The event-related spectral perturbation method using the newtimef function of $\mathrm{EEGLAB}^{54}$ will be used to compute the ERD power. The ERD power will be baseline corrected. Subsequently, the power will be averaged across all trials and converted to log power. Averaged ERD powers at electrode sites C3 (ipsilesional hemisphere, $\mathrm{IH}$ ) and $\mathrm{C} 4$ (contralesional hemisphere, $\mathrm{CH}$ ) will be extracted. Data from patients with right brain lesions will be flipped to ensure that C3 channels stand for IHs and $\mathrm{C} 4$ channels stand for $\mathrm{CHs}$.

For movement-related ERD, the power at C3 will be used for further analysis. For MVF-induced ERD, the powers at $\mathrm{IH}$ and $\mathrm{CH}$ during the movement phase will be extracted and an asymmetric index will be calculated with the following formula ${ }^{55}$ :

$$
\text { Asymmetric index }=(\text { IH ERD power })-(\text { CH ERD power })
$$

The difference of asymmetric indices under the mirror view and direct view will be used to evaluate MVF-induced sensorimotor ERD and used in a further analysis. A more negative value indicates more activation toward the ipsilesional sensorimotor area, during the mirror view condition, compared with the direct view condition. Mu-1 $(8-10 \mathrm{~Hz})$, mu-2 $(10-12 \mathrm{~Hz})$, beta-1 $(12-16 \mathrm{~Hz})$ and beta-2 $(16-30 \mathrm{~Hz})$ will be investigated separately. ${ }^{32}$ 


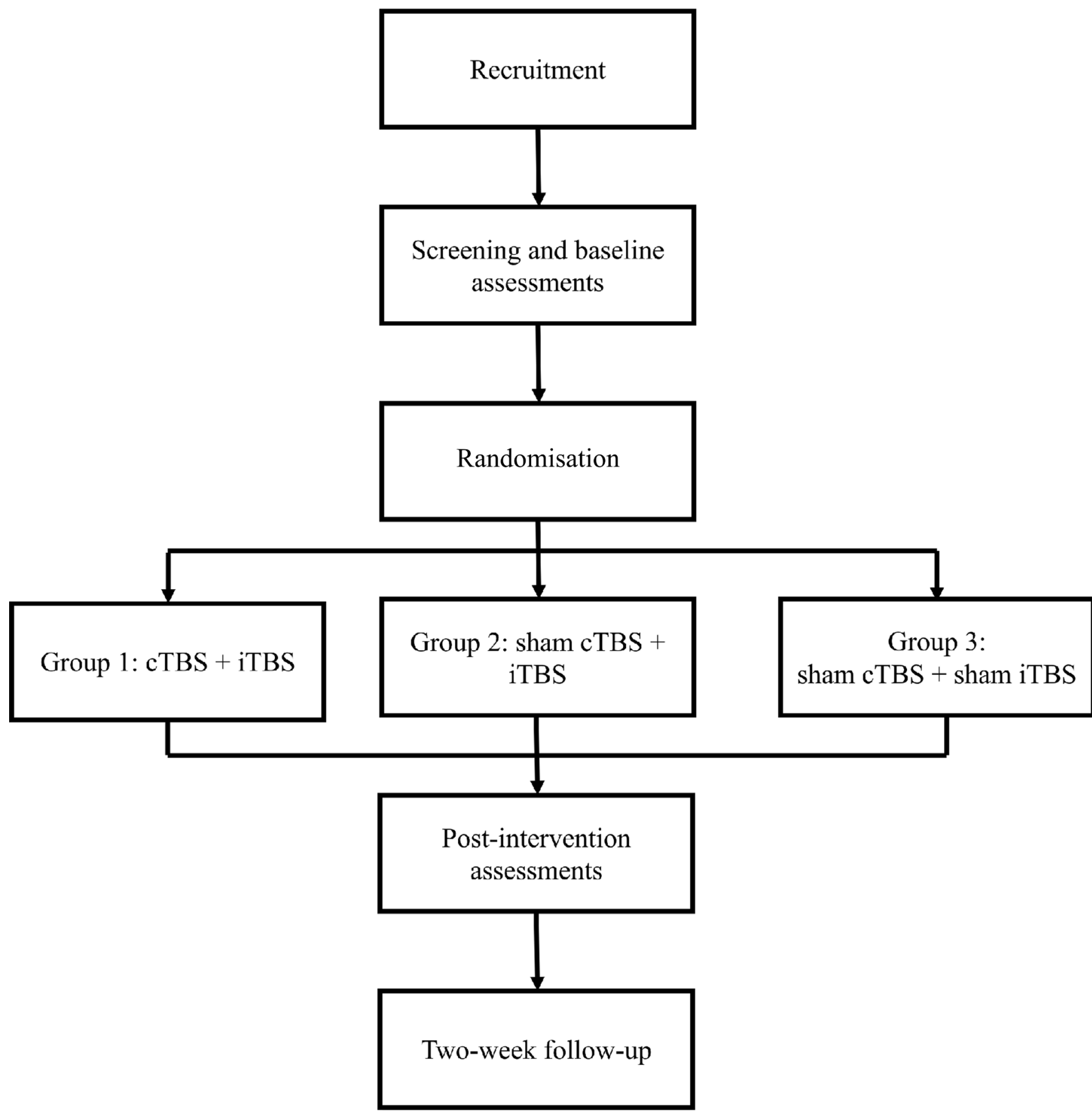

Figure 2 Flowchart of the proposed randomised controlled trial.

\section{Safety profile investigation}

A side-effects survey will be distributed on completion of each TBS session. See figure 3 for an overview of the proposed trial.

\section{Statistical analysis}

Statistical analysis will be performed using SPSS V.23.0. Demographic and baseline characteristics will be compared using analysis of variance (continuous and ordinal data) or $\chi^{2}$ tests (categorical data). A mixed-effects model with random intercepts and slopes will be used to detect any significant differences in the rate of change in motor outcomes and sensorimotor ERD among the three groups, because of its superiority in analysing repeated measures data and dataset with missing values. Any factor with significant between-group difference in the baseline will be included in the mixed-effects model as covariates. Group effects, time effects and group-by-time interaction effects will be included as fixed effects, and the random intercept and random slope of change in the dependent variables over time will be included as random effects. Between-group differences will be investigated using the interaction effects. Maximum likelihood estimation will 


\begin{tabular}{|l|c|c|c|c|c|}
\multicolumn{1}{|c|}{ Timepoint } & $\begin{array}{c}- \text { T1 } \\
\text { (Screening) }\end{array}$ & $\begin{array}{c}\text { T0 } \\
\text { (Baseline) }\end{array}$ & $\begin{array}{c}\text { T1 } \\
\text { (Mid) }\end{array}$ & $\begin{array}{c}\text { T2 } \\
\text { (Post) }\end{array}$ & $\begin{array}{c}\text { T3 } \\
\text { (Follow-up) }\end{array}$ \\
\hline Recruitment & $\mathrm{X}$ & & & \\
\hline Eligibility screening & $\mathrm{X}$ & &
\end{tabular}

Informed consent $\quad \mathrm{X}$

Randomisation

$$
\text { X }
$$

\section{Intervention}

Group 1: cTBS +

iTBS combined with

RAT

\section{Group 2: sham cTBS \\ + iTBS combined with RAT \\ Group 3: Sham cTBS \\ + sham iTBS \\ combined with RAT}

\begin{tabular}{|c|c|c|c|c|c|}
\hline \multicolumn{6}{|l|}{ Assessments } \\
\hline FMA-UE & $\mathrm{X}$ & & $\mathrm{X}$ & $\mathrm{X}$ & $\mathrm{X}$ \\
\hline ARAT & $X$ & & $\mathrm{X}$ & $X$ & $\mathrm{X}$ \\
\hline $\begin{array}{l}\text { Side-effects } \\
\text { questionnaire }\end{array}$ & & $\mathrm{X}$ & $X$ & $\mathrm{X}$ & \\
\hline Kinematic outcomes & & $\mathrm{X}$ & $\mathrm{X}$ & $\mathrm{X}$ & \\
\hline EEG & & $X$ & & $X$ & \\
\hline
\end{tabular}

Figure 3 Schedule of participant recruitment, assessments and intervention. ARAT, Action Research Arm Test; cTBS, continuous theta burst stimulation; EEG, electroencephalography; FMA-UE, Fugl-Meyer Assessment-Upper Extremity scores; iTBS, intermittent theta burst stimulation; RAT, robot-assisted training.

be chosen as the estimation method. The covariance structure is assumed to be unstructured. The level of significance will be set at $\mathrm{p}<0.05$. For post-hoc comparisons, the level of significance will be set at $\mathrm{p}<0.017$ after Bonferroni adjustment $(0.05 / 3 ; n=$ number of comparisons), for the comparison of interaction effects. Cohen's $\mathrm{d}$ will be calculated to determine the effect size of the change scores for the behavioural motor outcomes between groups. Immediate training effects (data from baseline to post-training) and the durability of training effects (data from post-training to follow-up) will be separately investigated with mixed-effect models. Frequency scores for each reported side effect and the percentage of participants who pass the MCID of the FMA-UE and ARAT will be compared using $\chi^{2}$ tests between the three groups.

\section{Patient and public involvement}

Patients will be invited to participate in this study via advertisements. Several self-help stroke organisations will be notified in order to promote the enrolment. The results of the evaluation can be released to participants on request. 


\section{ETHICS AND DISSEMINATION}

This RCT was registered on 24 July 2019 (https:/ / clinicaltrials.gov, see online supplementary section for trial registration data). The study has launched on 9 September 2019 and will continue for around a year. The study will be conducted in accordance with the principles of the Declaration of Helsinki. Written informed consent forms will be collected from each participant before the study begins (see a template of written consent form in online supplementary section). Any modifications to this study protocol will also be reviewed by the subcommittee. This study will only include participants who have given informed written consent and the confidentiality is assured. All original data will be kept in strictly private. During the study, written data will be stored in a safe place; after the study, all data will be input to a computer by the principle investigator and a backup of the data will be kept on a hard drive, which will be stored in a safe place. The input data will be double checked by another research assistant. Personal data will be discarded after 3 years. Due to the small expected sample size of this proof-of-concept study, a data monitoring committee was not deemed to be required and we will perform interim analyses when $50 \%$ of patients have been included and have completed the follow-up assessment. The results of this study will be presented at international conferences and sent to a peer-reviewed journal to be considered for publication.

Contributors JJZ and KNKF were involved in the conception and design of the research. JJZ wrote up the first draft of the research. KNKF reviewed and edited the manuscript. JJZ and KNKF approved the submission of the final version of the manuscript.

Funding Work of JJZ was supported by PhD studentships of The Hong Kong Polytechnic University.

Competing interests None declared.

Patient and public involvement Patients and/or the public were not involved in the design, or conduct, or reporting, or dissemination plans of this research.

Patient consent for publication Not required.

Ethics approval Ethical approval has been obtained from The Human Subjects Ethics Sub-committee, University Research Committee of The Hong Kong Polytechnic University (reference number: HSEARS20190718003).

Provenance and peer review Not commissioned; externally peer reviewed.

Open access This is an open access article distributed in accordance with the Creative Commons Attribution Non Commercial (CC BY-NC 4.0) license, which permits others to distribute, remix, adapt, build upon this work non-commercially, and license their derivative works on different terms, provided the original work is properly cited, appropriate credit is given, any changes made indicated, and the use is non-commercial. See: http://creativecommons.org/licenses/by-nc/4.0/.

ORCID iD

Jack Jiaqi Zhang http://orcid.org/0000-0002-4656-1909

\section{REFERENCES}

1 Fisicaro F, Lanza G, Grasso AA, et al. Repetitive transcranial magnetic stimulation in stroke rehabilitation: review of the current evidence and pitfalls. Ther Adv Neurol Disord 2019;12:1-17.

2 Rossi S, Hallett M, Rossini PM, et al. Safety, ethical considerations, and application guidelines for the use of transcranial magnetic stimulation in clinical practice and research. Clin Neurophysiol 2009;120:2008-39.
3 Suppa A, Huang Y-Z, Funke K, et al. Ten years of theta burst stimulation in humans: established knowledge, unknowns and prospects. Brain Stimul 2016;9:323-35.

4 Huang Y-Z, Rothwell JC. The effect of short-duration bursts of highfrequency, low-intensity transcranial magnetic stimulation on the human motor cortex. Clin Neurophysiol 2004;115:1069-75.

5 Chen Y-J, Huang Y-Z, Chen C-Y, et al. Intermittent theta burst stimulation enhances upper limb motor function in patients with chronic stroke: a pilot randomized controlled trial. BMC Neurol 2019;19:69.

6 Hsu Y-F, Huang Y-Z, Lin Y-Y, et al. Intermittent theta burst stimulation over ipsilesional primary motor cortex of subacute ischemic stroke patients: a pilot study. Brain Stimul 2013;6:166-74.

7 Talelli P, Wallace A, Dileone M, et al. Theta burst stimulation in the rehabilitation of the upper limb: a semirandomized, placebocontrolled trial in chronic stroke patients. Neurorehabil Neural Repair 2012;26:976-87.

8 Talelli P, Greenwood RJ, Rothwell JC. Exploring theta burst stimulation as an intervention to improve motor recovery in chronic stroke. Clin Neurophysiol 2007;118:333-42.

9 Chung SW, Hill AT, Rogasch NC, et al. Use of theta-burst stimulation in changing excitability of motor cortex: a systematic review and meta-analysis. Neurosci Biobehav Rev 2016;63:43-64.

10 Watanabe K, Kudo Y, Sugawara E, et al. Comparative study of ipsilesional and contralesional repetitive transcranial magnetic stimulations for acute infarction. J Neurol Sci 2018;384:10-14.

11 Ackerley SJ, Byblow WD, Barber PA, et al. Primed physical therapy enhances recovery of upper limb function in chronic stroke patients. Neurorehabil Neural Repair 2016;30:339-48.

12 Sung W-H, Wang C-P, Chou C-L, et al. Efficacy of coupling inhibitory and facilitatory repetitive transcranial magnetic stimulation to enhance motor recovery in hemiplegic stroke patients. Stroke 2013;44:1375-82.

13 Ackerley SJ, Stinear CM, Barber PA, et al. Priming sensorimotor cortex to enhance task-specific training after subcortical stroke. Clin Neurophysiol 2014;125:1451-8.

14 Meehan SK, Dao E, Linsdell MA, et al. Continuous theta burst stimulation over the contralesional sensory and motor cortex enhances motor learning post-stroke. Neurosci Lett 2011;500:26-30.

15 Zhang L, Xing G, Fan Y, et al. Short- and long-term effects of repetitive transcranial magnetic stimulation on upper limb motor function after stroke: a systematic review and meta-analysis. Clin Rehabil 2017;31:1137-53.

16 Schilberg L, Schuhmann T, Sack AT. Interindividual variability and Intraindividual reliability of intermittent theta burst stimulationinduced neuroplasticity mechanisms in the healthy brain. $J$ Cogn Neurosci 2017;29:1022-32.

17 Vernet M, Bashir S, Yoo W-K, et al. Reproducibility of the effects of theta burst stimulation on motor cortical plasticity in healthy participants. Clin Neurophysiol 2014;125:320-6.

18 Goldsworthy MR, Müller-Dahlhaus F, Ridding MC, et al. Inter-subject variability of LTD-like plasticity in human motor cortex: a matter of preceding motor activation. Brain Stimul 2014;7:864-70.

19 Cassidy JM, Gillick BT, Carey JR. Priming the brain to capitalize on metaplasticity in stroke rehabilitation. Phys Ther 2014;94:139-50.

20 Hassanzahraee M, Zoghi M, Jaberzadeh S. How different priming stimulations affect the corticospinal excitability induced by noninvasive brain stimulation techniques: a systematic review and meta-analysis. Rev Neurosci 2018;29:883-99.

21 Opie GM, Vosnakis E, Ridding MC, et al. Priming theta burst stimulation enhances motor cortex plasticity in young but not old adults. Brain Stimul 2017;10:298-304.

22 Mastroeni C, Bergmann TO, Rizzo V, et al. Brain-derived neurotrophic factor--a major player in stimulation-induced homeostatic metaplasticity of human motor cortex? PLoS One 2013;8:e57957-e57.

23 Murakami T, Müller-Dahlhaus F, Lu M-K, et al. Homeostatic metaplasticity of corticospinal excitatory and intracortical inhibitory neural circuits in human motor cortex. J Physiol 2012;590:5765-81.

24 Concerto C, Lanza G, Cantone M, et al. Repetitive transcranial magnetic stimulation in patients with drug-resistant major depression: a six-month clinical follow-up study. Int J Psychiatry Clin Pract 2015;19:252-8.

25 lyer MB, Schleper N, Wassermann EM. Priming stimulation enhances the depressant effect of low-frequency repetitive transcranial magnetic stimulation. J Neurosci 2003;23:10867-72.

26 Boyd LA, Hayward KS, Ward NS, et al. Biomarkers of stroke recovery: consensus-based core recommendations from the stroke recovery and rehabilitation roundtable. Neurorehabil Neural Repair 2017;31:864-76. 
27 Neuper C, Wörtz M, Pfurtscheller G. ERD/ERS patterns reflecting sensorimotor activation and deactivation. Prog Brain Res 2006;159:211-22.

28 Shiner CT, Tang H, Johnson BW, et al. Cortical beta oscillations and motor thresholds differ across the spectrum of post-stroke motor impairment, a preliminary MEG and TMS study. Brain Res 2015;1629:26-37.

29 Rossiter HE, Boudrias M-H, Ward NS. Do movement-related beta oscillations change after stroke? J Neurophysiol 2014;112:2053-8.

30 Bartur G, Pratt H, Frenkel-Toledo S, et al. Neurophysiological effects of mirror visual feedback in stroke patients with unilateral hemispheric damage. Brain Res 2018;1700:170-80.

31 Bartur G, Pratt H, Dickstein R, et al. Electrophysiological manifestations of mirror visual feedback during manual movement. Brain Res 2015;1606:113-24.

32 Zhang JJ, Fong KNK. Enhancing mirror visual feedback with intermittent theta burst stimulation in healthy adults. Restor Neurol Neurosci 2019;37:483-95.

33 Dionísio A, Gouveia R, Duarte IC, et al. Continuous theta burst stimulation increases contralateral mu and beta rhythms with arm elevation: implications for neurorehabilitation. J Neural Transm 2020;127:17-25.

34 Tremblay S, Rogasch NC, Premoli I, et al. Clinical utility and prospective of TMS-EEG. Clin Neurophysiol 2019;130:802-44.

35 Chan A-W, Tetzlaff JM, Altman DG, et al. Spirit 2013 statement: defining standard protocol items for clinical trials. Ann Intern Med 2013;158:200-7.

36 Bernhardt J, Hayward KS, Kwakkel G, et al. Agreed definitions and a shared vision for new standards in stroke recovery research: the stroke recovery and rehabilitation roundtable Taskforce. Int J Stroke 2017;12:444-50.

37 Fong $\mathrm{K}, \mathrm{Ng} \mathrm{B}$, Chan D, et al. Development of the Hong Kong version of the functional test for the hemiplegic upper extremity (FTHUE-HK). Hong Kong Journal of Occupational Therapy 2004;14:21-9.

38 Jin M, Zhang Z, Bai Z, et al. Timing-Dependent interaction effects of tDCS with mirror therapy on upper extremity motor recovery in patients with chronic stroke: a randomized controlled pilot study. $J$ Neurol Sci 2019;405:116436.

39 Fong KN, Lo PC, Yu YS, et al. Effects of sensory cueing on voluntary arm use for patients with chronic stroke: a preliminary study. Arch Phys Med Rehabil 2011:92:15-23.

40 Chu L, Pei C, Ho M, et al. Validation of the abbreviated mental test (Hong Kong version) in the elderly medical patient. Hong Kong Med J 1995;1:207-11.

41 Bohannon RW, Smith MB. Interrater reliability of a modified Ashworth scale of muscle spasticity. Phys Ther 1987;67:206-7.
42 Zigmond AS, Snaith RP. The hospital anxiety and depression scale. Acta Psychiatr Scand 1983;67:361-70.

43 Jensen CV. A computer program for randomizing patients with near-even distribution of important parameters. Comput Biomed Res 1991;24:429-34.

44 Qian Q, Nam C, Guo Z, et al. Distal versus proximal - an investigation on different supportive strategies by robots for upper limb rehabilitation after stroke: a randomized controlled trial. J Neuroeng Rehabil 2019;16:64.

45 Hu X-L, Tong RKY, Ho NSK, et al. Wrist rehabilitation assisted by an Electromyography-Driven neuromuscular electrical stimulation robot after stroke. Neurorehabil Neural Repair 2015;29:767-76.

46 Dieler AC, Dresler T, Joachim K, et al. Can intermittent theta burst stimulation as add-on to psychotherapy improve nicotine abstinence? results from a pilot study. Eur Addict Res 2014;20:248-53.

47 Kwakkel G, Lannin NA, Borschmann K, et al. Standardized measurement of sensorimotor recovery in stroke trials: consensusbased core recommendations from the stroke recovery and rehabilitation roundtable. Int J Stroke 2017;12:451-61.

48 Page SJ, Levine P, Hade E. Psychometric properties and administration of the wrist/hand subscales of the Fugl-Meyer assessment in minimally impaired upper extremity hemiparesis in stroke. Arch Phys Med Rehabil 2012;93:2373-6.

49 Van der Lee JH, De Groot V, Beckerman H, et al. The intra- and interrater reliability of the action research arm test: a practical test of upper extremity function in patients with stroke. Arch Phys Med Rehabil 2001;82:14-19.

50 Chan IHL, Fong KNK, Chan DYL, et al. Effects of arm weight support training to promote recovery of upper limb function for subacute patients after stroke with different levels of arm impairments. Biomed Res Int 2016;2016:1-9.

51 Rossiter HE, Borrelli MR, Borchert RJ, et al. Cortical mechanisms of mirror therapy after stroke. Neurorehabil Neural Repair 2015;29:444-52.

52 Lee H-M, Li P-C, Fan S-C. Delayed mirror visual feedback presented using a novel mirror therapy system enhances cortical activation in healthy adults. J Neuroeng Rehabil 2015;12:56.

53 Delorme A, Makeig S. EEGLAB: an open source toolbox for analysis of single-trial EEG dynamics including independent component analysis. J Neurosci Methods 2004;134:9-21.

54 Makeig S. Auditory event-related dynamics of the EEG spectrum and effects of exposure to tones. Electroencephalogr Clin Neurophysiol 1993;86:283-93.

55 Fong KN, Ting $\mathrm{KH}$, Chan CC, et al. Mirror therapy with bilateral arm training for hemiplegic upper extremity motor functions in patients with chronic stroke. Hong Kong Med J 2019;25:30-4. 